\title{
Responsabilités Neuroéthiques
}

\author{
Eric Racine, Judy Illes
}

RÉSUMÉ: Les neurosciences représentent un secteur dynamique de la recherche biomédicale où des responsabilités neuroéthiques pour les chercheurs émergent. Cet article accompagne la version anglaise publiée dans ce numéro du Canadian Journal of Neurological Sciences. Nous présentons des avancées récentes en neuroéthique à l'aide de trois cas: (1) la découverte fortuite d'anomalies en neuroimagerie; (2) la création de neurotechnologies pouvant conduire à l'amélioration de la cognition humaine et (3) la communication responsable des résultats de recherche. Nous présentons et discutons ensuite d'un cadre multidimensionnel de responsabilités neuroéthiques pouvant aider à aborder de front ces enjeux. Ce cadre réitère le caractère fondamental de l'intégrité scientifique, met de l'avant les responsabilités sociales à l'égard de l'usage éventuel des connaissances scientifiques et met à l'avant-plan la réflexion autocritique dans la recherche et la formation des chercheurs.

\begin{abstract}
Neuroscience represents a dynamic area of biomedical research where neuroethical responsibilities for researchers are emerging. This paper is the companion piece to the English-language one also published in this issue of the Canadian Journal of Neurological Sciences. It serves as a review of recent advances in neuroethics through the lens of three cases: (1) incidental finding of anomalies in neuroimaging research; (2) creation of neurotechnologies that can lead to cognitive enhancement, and (3) responsible communication of research results. We propose and discuss a multidimensional framework of neuroethical responsibilities to help tackle these issues. The framework reiterates the fundamental role of scientific integrity, puts in the foreground social responsibilities pertaining to the eventual use of neuroscience knowledge, and highlights self-reflection in research and training of researchers.
\end{abstract}

Can. J. Neurol. Sci. 2006; 33: 260-268

Tout comme la recherche en génétique, les neurosciences avancent rapidement. Contrairement à la génétique, les sciences du cerveau n'ont cependant pas fait jusqu'à maintenant l'objet d'une réflexion éthique systématique et interdisciplinaire. ${ }^{1}$ Or, de nouvelles applications de neurotechnologies de pointe soulèvent avec acuité des enjeux éthiques comme, par exemple, en matière de consentement des patients vulnérables ${ }^{2}$ et d'allocation des resources. ${ }^{3}$ Des tentatives d'applications non médicales de la neuroimagerie dans le domaine judiciaire ${ }^{4}$ et dans le domaine de l'éducation ${ }^{5}$ retiennent l'attention du public et provoquent des débats au sein des neuroscientifiques et des bioéthiciens. Il y a également une discussion grandissante de l'éthique de la neuroimagerie dans des magazines à grand tirage ${ }^{6}$ ainsi que dans des revues scientifiques prestigieuses. ${ }^{7,8}$ Nombre d'enjeux sont présentement discutés et requièrent des réponses éthiques proactives (Tableau 1). Compte tenu des avancées récentes, les neurosciences représentent donc un secteur de recherche où de nouvelles responsabilités pour les chercheurs émergent.

Cet article présente le contexte dans lequel se présentent les enjeux éthiques émergents des neurosciences et les défis qui en découlent sur le plan de la responsabilité des chercheurs. Trois cas illustrent ce contexte : (1) la découverte fortuite d'anomalies en neuroimagerie; (2) la création de neurotechnologies pouvant conduire à l'amélioration de la cognition humaine et (3) la communication responsable des résultats de recherche. Suite à une analyse éthique des enjeux soulevés, nous proposons un cadre multidimensionnel de responsabilités neuroéthiques qui réitère le caractère fondamental de l'intégrité scientifique, souligne les responsabilités sociales associées à l'usage éventuel des connaissances neuroscientifiques et met de l'avant l'importance de la réflexion autocritique dans la conduite de la recherche et dans la formation des chercheurs. Conscients que ni notre analyse ni nos recommandations ne sont exhaustives ou finales, nous espérons provoquer une discussion sur les enjeux éthiques des neurosciences. L'accent mis sur les enjeux émergents s'appuie sur la tradition de réflexion en neurologie et en psychiatrie ainsi que la valeur des lignes directrices en matière d'éthique de la recherche.

Du The Neuroethics Imaging Group (ER), Department of Radiology (JI), Stanford Center for Biomedical Ethics, Palo Alto, California, USA.

ReÇu DeCEMber 14, 2005. ACCePté DANS la fORME FinAle April 22, 2006. Réimprimez demande à: Eric Racine, Neuroethics Research Unit, Institute de recherches cliniques de Montreal (IRCM), Office 1535, 110, Pine Avenue West, Montreal, Quebec H2W 1R7. 


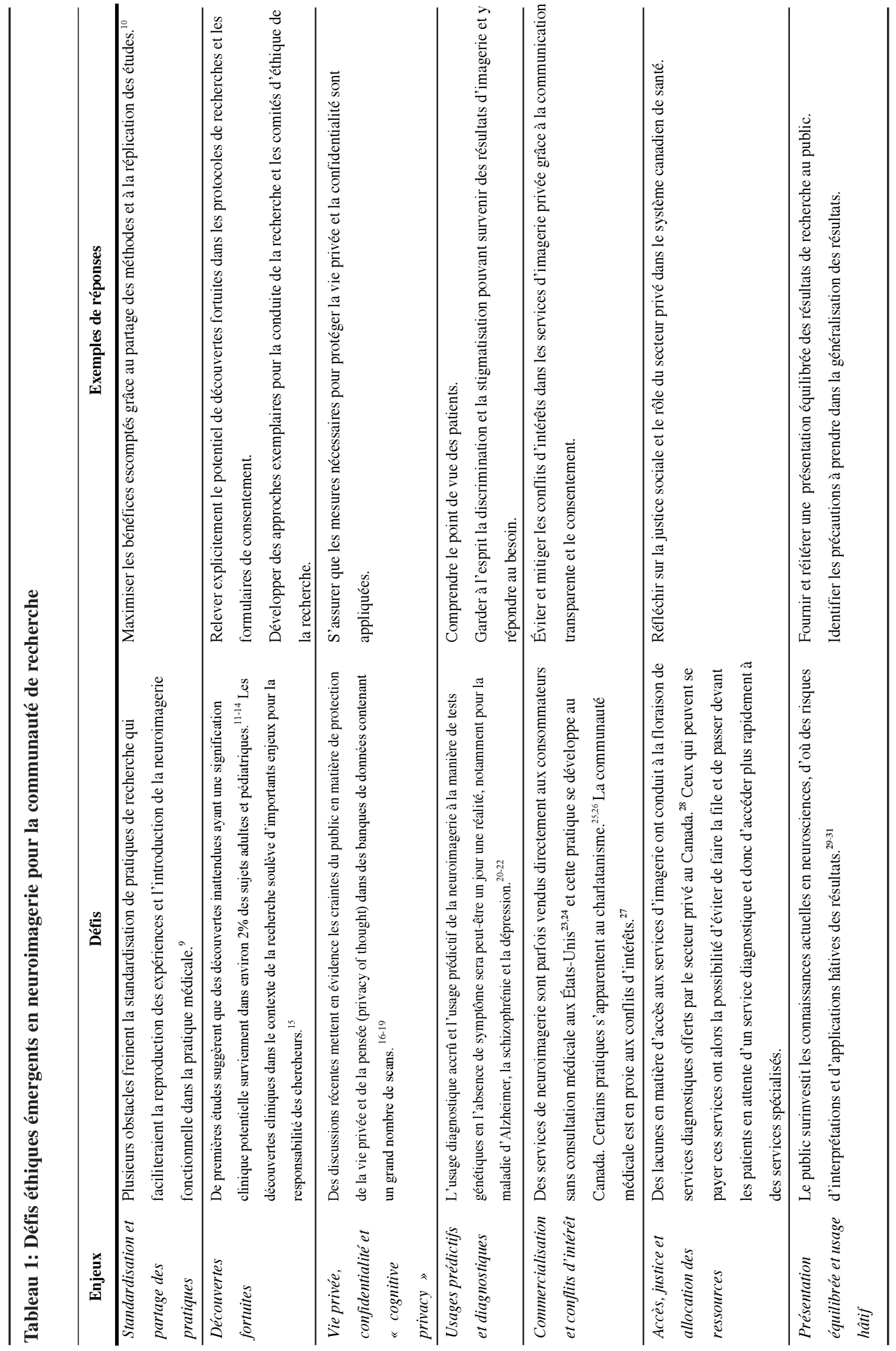




\section{Premier Cas \\ DECOUVRIR FORTUITEMENT DES ANOMALIES DANS LE CONTEXTE DE LA RECHERCHE}

Les puissantes propriétés des techniques de neuroimagerie créent des défis spécifiques pour les chercheurs et la conduite responsable de la recherche lorsque des anomalies sont mises en évidence chez des sujets sains. Ces découvertes ont été nommées «découvertes fortuites» (ou incidental findings) et peuvent être définies comme des anomalies insoupçonnées avant le scan cérébral et ayant potentiellement une signification clinique sans pour autant que ces anomalies ne soient. ${ }^{32}$ Des cas réels de découvertes fortuites en neuroimagerie illustrent certains des défis. Une jeune étudiante en médecine de l'Université Stanford a relaté son expérience suite à la découverte fortuite d'une malformation artério-veineuse (voir: http//neuroethics. stanford.edu: The Case of SH). ${ }^{33}$ Heureusement son histoire touchante s'est terminée avec des chirurgies réussies. Elle poursuit présentement sa formation en médecine, transformée par cette expérience de ç titre de patiente-étudiante. Un correspondant anonyme a décrit récemment dans Nature comment une découverte fortuite d'une tumeur cérébrale trouvée dans le cadre d'un projet de recherche a complètement modifié sa trajectoire de vie. Après qu'il ait divulgué la découverte fortuite à un assureur, il s'est vu refuser une couverture d'assurance emploi privée. ${ }^{34}$

La gestion des découvertes fortuites pose avec acuité des questions importantes quant à la responsabilité des chercheurs et celle des établissements où se déroulent les études de neuroimagerie. Des études préliminaires suggèrent que de telles découvertes fortuites, bien que rares, surviennent sur une base régulière dans environ $2 \%$ de la population adulte et pédiatrique. ${ }^{11-13}$ Ces données excèdent celles de certaines études populationnelles telle que le registre américain des tumeurs cérébrales. ${ }^{14}$ Bien que la majorité de ces découvertes fortuites se révèlent bénignes suite à un examen plus approfondi, leur présence dans un environnement de recherche soulève avec acuité des questions touchant à la responsabilité des chercheurs. ${ }^{15}$ Les sources de la variabilité et de l'incidence de ces anomalies restent à être examinées. De plus, il y a peu de données quant aux coûts et aux bénéfices de faux résultats positifs. Par contre, ces anomalies ne peuvent être simplement ignorées comme des chercheurs américains, certaines décisions de tribunaux ${ }^{35-37}$ et un consensus émergent le reflètent. ${ }^{38}$ Cette question des découvertes fortuites souligne la valeur d'attitudes fondées sur la sollicitude à l'égard des sujets de recherche.

\section{DeUXième CaS \\ Améliorer la COGNition hUMAINE}

Les bénéfices escomptés des neurosciences sont intimement associés au traitement des maladies neurologiques et psychiatriques. Par contre, une possibilité plus controversée point à l'horizon. Il s'agit de l'amélioration (enhancement) de la cognition, des émotions et de l'attention grâce à diverses formes de neurotechnologies. Déjà il y a une quinzaine d'années, l'usage croissant de Prozac a suscité un vif débat sur la possibilité de se sentir «better than well». ${ }^{39}$ Aujourd'hui, les possibilités d'amélioration pharmacologique et les usages liés au mode de vie se multiplient au même moment où le marketing des compagnies pharmaceutiques s'intensifie et se ramifie. ${ }^{40,41}$ De plus, l'usage d'agents neuropharmacologiques crôit et représente une dépense de santé grandissante au Canada. ${ }^{42-44}$

Le cas de l'amélioration de la cognition humaine, où science et spéculation s'enchevêtrent, permet d'illustrer la valeur d'une discussion prospective sur les responsabilités sociales élargies au sein de la communauté des neuroscientifiques quant aux conséquences éventuelles d'applications ayant pour fin l'amélioration des performances cognitives. Le tableau 2 (voir la page 4) présente les résultats de travaux récents qui pourraient conduire à des opportunités d'amélioration neuropharmacologique (par ex.: capacités de concentration, de mémoire et d'éveil). Nous avons également identifié des enjeux éthiques associés à ces possibilités tels que la clarification des limites des neurotechnologies améliorantes et la prédiction des conséquences de tels usages.

Sources d'immenses espoirs thérapeutiques, les avancées de la neuropharmacologie pourraient conduire à une «neurologie cosmétique» (cosmetic neurology). ${ }^{40}$ Les enjeux éthiques y étant associés sont imposants. L'amélioration pourrait accentuer des questions de justice distributive et remettre en question le sens de l'intervention médicale et de la médecine dans les sociétés modernes. ${ }^{56}$ La coercition représente un autre enjeu considérable si l'on tient compte à la fois des précédents d'usage de produits améliorants tels que les amphétamines dans le contexte militaire américain ainsi que des taux élevés d'usage du Ritalin chez les écoliers en particulier chez les garçons. ${ }^{41}$ Il reste en outre à examiner toutefois si l'amélioration d'une fonction cérébrale est réellement possible et quelles en seraient les conséquences médicales et sociales.

Bien que la neuropharmacologie pourrait bien être le secteurclef en matière d'amélioration, il faut noter que d'autres secteurs de recherche conduisent à des neurotechnologies ayant le potentiel d'amélioration. Par exemple, l'espoir de développer une interface fonctionnelle entre les tissus neuronaux et les composantes informatiques anime la recherche depuis plusieurs décennies. ${ }^{57}$ De récentes avancées laissent croire que le rêve d'une telle interface fonctionnelle neuro-ordinateur se rapproche de la réalité ${ }^{58-60}$ bien que plusieurs grands défis subsistent. Le traitement de la maladie de Parkinson constitue l'un des plus grands succès médicaux de ces interfaces. ${ }^{61}$ L' usage d'implants cérébraux pourrait maintenant s'étendre. ${ }^{2}$ Par exemple, un groupe torontois a publié récemment des résultats suggérant l'efficacité de neurostimulateurs (neurostimulation à haute fréquence) dans le traitement de la dépression sévère. ${ }^{62}$ Nicolelis, un chercheur en vue dans cette communauté, a suggéré que ces technologies pourraient provoquer «a revolution in the way future generations interact with computers, virtual objects and remote environments, by allowing never-before-experienced augmentation of perceptual, motor and cognitive capabilities». ${ }^{57}$ Certains se demandent s'il sera un jour possible d'ajouter des modalités sensorielles ou d'accélérer le traitement de l'information et des processus cognitifs chez des individus sains. ${ }^{63}$ En ce moment, le caractère invasif de ces interfaces limite ces usages améliorants, mais qu'adviendra-t-il si les interfaces deviennent moins invasives, encore plus sécuritaires et davantage acceptées par la société?

Certains s'intéressent déjà sérieusement à de tels usages améliorants. Des représentants de la Defense Advanced Research 


\title{
Tableau 2: Exemples de possibles agents neuropharmacologiques améliorants
}

\author{
Possibilités d'amélioration et enjeux associés
}

Amélioration des capacités de concentration

Le public s'inquiète de l'usage éthique du methylphenidate (Ritalin) dans le traitement du trouble de déficit de l'attention/hyperactivité (TDA/H). ${ }^{45}$ Des recherches suggèrent que le Ritalin peut améliorer les performances normales dans diverses tâches exécutives et le fonctionnement de la mémoire de travail. ${ }^{46,47}$ Une étude indique que « l'usage récréatif » du methylphenidate s'élève $17 \%$ chez 283 répondants dans un collège américain d'arts libéraux. ${ }^{48}$

Amélioration de la mémoire

Des agents pharmacologiques pouvant pallier aux déficits de la cognition et de la mémoire, principalement ceux causés par des maladies neurodégénératives telles que la maladie d'Alzheimer pourraient possiblement se prêter à l'amélioration des performances cognitives. ${ }^{49,50}$ Une étude rapporte que Donepezil, un inhibiteur de l'acétylcholisestérase présentement utilisé dans le traitement de la maladie d'Alzheimer, améliore les performances des pilotes de lignes commerciales dans le cadre de simulations de vols. ${ }^{51}$

Élimination des souvenirs

Une étude pilote suggère qu'un bloqueur du récepteur ß-adrénerique (Propranolol) pourrait prévenir ou affaiblir la consolidation de souvenirs indésirables associés au stress post-traumatique. ${ }^{52}$ Amélioration de l'éveil

90\% des prescriptions de Provigil sont en dérogation de directives (off-label) et certains usages rapportés ont pour objectif l'amélioration des performances sportives ou remédier aux décalages horaires. $^{53}$

\section{Exemples de réponses}

- Clarifier les limites scientifiques et éthiques des neurotechnologies améliorantes.

- Déterminer si des améliorations consistant à combattre le sommeil ou des souvenirs douloureux doivent faire partie de la médecine.

- Dans le cas du Ritalin en particulier, tenir compte de la vulnérabilité des enfants et des adolescents, notamment sur le plan de la coercition, des pressions sociales et de l'allocation des ressources.

- Identifier les causes des usages améliorants et les aspirations auxquelles ils répondent.

- Clarifier les usages acceptables des neurotechnologies améliorantes afin d'éviter les abus et d'assurer les meilleurs usages éthiquement possibles.

- Prévoir les risques biologiques, psychologiques et sociales d'une augmentation des heures d'éveil ${ }^{54}$ et d' autres formes d'amélioration. ${ }^{55}$

Projects Agency, une agence qui finance plusieurs grands projets d'interfaces neuro-ordinateurs aux États-Unis ont confirmé que leurs intentions consistent à éventuellement améliorer les performances du personnel militaire, par exemple, en permettant de surveiller l'activité cérébrale du personnel. ${ }^{64,65}$ Une étude suggère que d'autres techniques de neurostimulation telles que la stimulation transcrânienne déjà utilisée pour le traitement de la dépression au Canada pourrait peut-être servir à améliorer de manière temporaire et réversible les performances cognitives. ${ }^{66}$

Hors du contexte de l'amélioration, les représentations publiques révèlent des préoccupations quant à l'approbation rapide de nouveaux usages de neurostimulateurs et de l'influence d'intérêts conflictuels étant donné que les normes de la Food and Drug Administration qui encadrent l'approbation d'appareils (devices) diffèrent des normes en place pour l'approbation des produits pharmaceutiques (drugs). ${ }^{67}$ Les compagnies d'appareils médicaux pourraient être intéressées à élargir les usages et les ventes en utilisant des stratégies parfois douteuses. Par exemple, l'une des principales entreprises d'équipements médicaux a été accusée en 2003 de verser des ristournes aux neurochirurgiens qui réalisaient des interventions de pointe (spinal fusion surgery). ${ }^{3}$ Cet exemple illustre que le processus de développement des neurotechnologies et les impératifs associés à leur commercialisation entrent parfois en conflit avec les visées de la médecine, particulièrement là où un système public de santé défraie une partie du coût des médicaments. ${ }^{68}$ On peut s'attendre à des enjeux semblables si l'amélioration neurotechnologique devient plus répandue conformément à une 
logique marchande. De fait, des précédents suggèrent que l'interaction entre les pratiques d'amélioration et les usages liés au mode de vie peuvent grandement compliquer l'analyse des enjeux éthiques des neurotechnologies. ${ }^{41,69,70}$

La participation des chercheurs aux discussions actuelles et futures sur l'amélioration s'avère essentielle pour qu'un débat public éclairé puisse avoir lieu. De plus, les prétentions à l'amélioration peuvent s'avérer une utopie ayant des conséquences néfastes insoupçonnées pour ses adeptes. Si de telles illusions ne sont pas dissipées par les chercheurs, nos sociétés risquent de se confronter à des abus fondés sur de mauvaises informations. ${ }^{50}$ Ainsi, dans un contexte où l'usage de certaines neurotechnologies avec un but explicite d'amélioration gagne du terrain, il devient primordial que la communauté neuroscientifique participe à des discussions proactives quant aux usages des technologies et leurs conséquences sociales élargies.

\section{Troisième Cas \\ Communiquer Responsablement Les RÉSultats DE RECHERCHE}

Les chercheurs doivent partager leurs résultats et participer au transfert des connaissances afin que le public soit informé des recherches en neurosciences et puisse faire bon usage des produits qui en découlent. Lorsque le financement de la recherche provient en partie de fonds publics, il s'ensuit une obligation accrue de partager les résultats ne serait-ce que pour assurer la libre circulation des connaissances. En communiquant les fruits de leur travail, les chercheurs courent cependant le risque que des incompréhensions et des interprétations erronées conduisent à des usages malencontreux.

L'histoire ancienne et récente des neurosciences révèle des situations où certaines interprétations troublantes furent véhiculées, parfois avec des conséquences dramatiques. Par exemple, la popularisation de la psychochirurgie ${ }^{71}$ provient en partie de comptes rendu optimistes retrouvés dans la presse écrite. ${ }^{72}$ Bien qu'elles furent toujours contestées par des scientifiques prudents, les thèses phrénologiques et leurs applications dans les domaines de l'éducation, de la religion, du droit et de la santé ${ }^{73}$ captivaient certains scientifiques ainsi qu'une partie du public qui fréquentaient les cabinets de phrénologie. Rappelons ici que Gall, le père de la phrénologie, souhaitait introduire l'analyse des bosses du crâne afin d'évaluer les risques de récidives des inculpés. ${ }^{74}$ Un autre exemple rappelant nos débats neuroéthiques actuels : au début du siècle dernier en Allemagne, des techniques pseudo-scientifiques de détection de l'activité électrique du cerveau telles que la diagnoscopie fascinent le public. Les théories de l'harmonie cérébrale et de la caractérologie, disséminées par des neuroscientifiques aussi influents que Cécile et Karl Vogt, promettaient de transformer ce type de techniques en outils de gestion sociale et d'orientation professionnelle. Au moment, où l'économie allemande périclitait dramatiquement suite à la Première Guerre Mondiale, il y avait un besoin politique d'obtenir des moyens efficaces pour gérer les ressources humaines et identifier les bons candidats dans le secteur de l'emploi. ${ }^{75}$ Ainsi, comme ces exemples l'illustrent, certains espoirs de transfert technologique qui nous semblent aujourd'hui simplistes se sont appuyées sur des interprétations emportées des neurosciences et de la fonction cérébrale.

Les croyances du public et l'interprétation des connaissances des chercheurs interagissent avec l'environnement politique et social élargi comme l'histoire le montre. Aujourd'hui, la volonté de guérir les maladies humaines renforce les attentes à l'égard des technologies médicales. Par conséquent, les tentatives d'appliquer rapidement les connaissances neuroscientifiques foisonnent. Par exemple, dans les années quatre-vingt-dix, les média ont popularisé les résultats d'une étude suggérant que les capacités intellectuelles des très jeunes enfants pouvaient être améliorées suite à la simple écoute d'enregistrements de musique classique. ${ }^{76}$ Cette étude avait été réalisée en fait sur des adultes et fut difficilement reproduite. Néanmoins, elle a conduit certains États américains à distribuer gratuitement des enregistrements sonores à tous les nouveaux-nés en dépit du fait que cette intervention de santé publique s'appuyait sur des interprétations audacieuses du développement cérébral et de la synaptogenèse. ${ }^{31}$ Autre exemple : aujourd'hui on peut lire dans les médias que les résultats d'études de neuroimagerie fournissent de nouvelles bases pour des pratiques sociales aussi diversifiées que le marketing, l'éducation et l'éthique. Ces études sont souvent réalisées sur un petit nombre de sujets et conformément à des devis expérimentaux singuliers. Elles ont donc des limites mais celles-ci sont peu rapportées dans la presse écrite. ${ }^{29}$ Ajoutant au défi de la communication responsable, un sondage portant sur les connaissances neuroscientifiques du public suggère que les méthodes de neuroimagerie sont parmi les concepts les moins bien compris du public. ${ }^{77}$ Ainsi, on voit donc que l'interprétation responsable des résultats n'est pas seulement un défi historique mais aussi bien contemporain. Les bénéfices attendus doivent êtres situés dans le contexte des risques associés au transfert des connaissances. Les divers compromis possibles à cet égard soulignent les responsabilités civiques et démocratiques des recherches ainsi que la valeur de la réflexion autocritique sur les limites actuelles des neurosciences tout en tenant compte d'événements historiques.

\section{DISCUSSION}

\section{Un cadre multidimensionnel de responsabilités neuroéthiques}

Notre analyse de trois cas provenant des neurosciences contemporaines suggère qu'il y a des défis qui se renouvellent sur le plan de la responsabilité des chercheurs. Nous proposons et discutons d'un cadre général de responsabilités neuroéthiques en réponse aux défis émergents (Tableau 3, inspiré de Racine ${ }^{78}$ et Illes, Racine et $\operatorname{Kirschen}^{10}$ ). Nous espérons que ce cadre provoquera une discussion sur les diverses responsabilités des neuroscientifiques, leur participation au débat public ainsi que la signification d'être un chercheur aux aguets de l'éthique dans le contexte actuel de la recherche.

\section{Responsabilité scientifique et intégrité}

\section{Réitérer l'importance fondamentale de la rigueur scientifique}

Les pressions actuelles poussent les chercheurs tout domaine confondu à des sommets de productivité scientifique mais cette mouvance pourrait aussi avoir des conséquences néfastes sur la recherche. ${ }^{79,80}$ Une étude publiée récemment dans Science suggère que des fautes professionnelles graves sont commises 
par une proportion non négligeable de scientifiques débutants et chevronnés. ${ }^{81}$ Les causes de ces phénomènes sont probablement profondes et touchent d'une manière ou d'une autre tous les chercheurs. On peut se rappeler ici la formule employée par l'exprésident du Comité Consultatif National d'Éthique en France, Jean Bernard, illustre médecin et scientifique : «ce qui n'est pas scientifique, n'est pas éthique». La rigueur et l'intégrité sont d'ailleurs une exigence fondamentale inscrite dans les lignes directrices du Council for International Organization of Medical Sciences.

L'intégrité scientifique implique également une collaboration accrue des chercheurs pour favoriser le développement de connaissances valides et reproductibles. ${ }^{82}$ Les bénéfices escomptés peuvent découler plus rapidement d'efforts concertés que d'efforts faiblement ou aucunement coordonnés. De nombreux obstacles peuvent freiner cette démarche : la faible disponibilité du financement pour la réplication formelle des études, pour la conservation de larges banques de données qui permettent de consolider les connaissances ${ }^{83}$ ou pour le partage de logiciels d'analyse utilisés pour analyser les données. ${ }^{9}$ Ces défis mettent en évidence l'importance de mettre à l'oeuvre la responsabilité scientifique.

Responsabilité-sollicitude

Considérer les intérêts des sujets humains dans un environnement de recherche de plus en plus complexe

La recherche qui est effectuée sur des sujets humains repose sur la bonne volonté de sujets de recherche. Dans le contexte des découvertes fortuites ou de la gestion de données confidentielles, il y a une tension entre le sujet, instrument de la recherche, et le sujet pris comme personne. Une attitude qui reconnaît les intérêts des sujets s'avérera un service au public et à la science.

\section{Responsabilité civique et démocratique}

Opter pour une vision large des conséquences et des implications sociales des neurosciences

Le public est en droit de s'attendre à la reconnaissance de sa participation au financement de la recherche. Des expériences heureuses de communication multidirectionnelle permettant aux

Tableau 3: Cade de responsabilités neuroéthiques

Responsabilités

\begin{tabular}{ll}
\hline Responsabilité scientifique & Réitérer l'importance fondamentale de la \\
et intégrité & rigueur scientifique.
\end{tabular}

\section{Exemples de réponses}

Validation des méthodes et coordination des collaborations multicentriques pour l'usage de nouvelles neurotechnologies.

Identifier les intérêts des sujets dans la gestion des découvertes fortuites.

dans un environnement de recherche de

plus en plus complexe.

Opter pour une vision large des

conséquences et des implications sociales

des neurosciences.
Présenter une vision équilibrée des mérites et des limites des neurotechnologies lors d'interactions avec les médias.

\section{Responsabilité prospective Participer aux discussions sur l'avenir des Participation aux réflexions éthiques sur les neurosciences et de leurs applications. possibilités d'usages améliorants des neurotechnologies.}

Réflexion autocritique Réfléchir sur les précédents.


chercheurs d'échanger avec le public ont lieu au Canada et ailleurs. ${ }^{84,85}$ Cependant, le transfert de connaissances ne va pas toujours de soi et de nombreuses difficultés peuvent surgir pour le chercheur souhaitant partager ses résultats et exercer une responsabilité civique et démocratique. Tel que nous l'avons mentionné précédemment, le public n'a pas toujours une compréhension approfondie des connaissances présentées. L'usage de la neuroimagerie à titre de preuve dans le droit criminel pour obtenir la clémence du jury ${ }^{86,87}$ ainsi que l'usage de la neuroimagerie pour sonder les préférences des consommateurs $^{8}$ sont deux exemples où une compréhension publique restreinte conduit à un transfert hâtif de technologie. Historiquement, des torts sont survenus d'applications rapides des connaissances et d'interprétations intempestives. Opter pour une vision large des conséquences et des implications sociales des neurosciences peut soutenir la clarification des limites des connaissances afin de donner un juste aperçu des bénéfices escomptés.

\section{Responsabilité prospective}

Participer aux discussions sur l'avenir des neurosciences et de leurs applications

Il $\mathrm{y}$ a des situations où science et science-fiction s'enchevêtrent. L'exemple de l'amélioration de la cognition grâce aux neurotechnologies en constitue un exemple. Les chercheurs peuvent parfois avoir des réserves quant aux applications projetées mais ils doivent demeurer ouverts aux scénarios éloignés, exercer une responsabilité proactive et considérer les conséquences lointaines de leurs actions. Le débat sur l'amélioration a présentement cours. Les chercheurs peuvent en profiter pour construire une neuroscience davantage informée par l'éthique.

\section{Réflexion autocritique}

\section{Réfléchir sur les précédents}

Une dimension de la responsabilité scientifique apparaît en filigrane de toutes les autres : la nécessité d'une réflexion autocritique sur ses propres travaux de recherche. Des précédents historiques illustrent comment des chercheurs en neurosciences ont pu soutenir les gestes les plus infâmes comme ceux ayant conduit à l'extermination des plus vulnérables dans le triste épisode du troisième Reich allemand. ${ }^{88}$ Face à cette sombre inhumanité, il y a l'illustre histoire des neurosciences. De grands neuroscientifiques ont marqué l'histoire de l'humanité. Leurs travaux ont changé des vies en favorisant une compréhension approfondie du système nerveux et en développant des thérapies et des attitudes compatissantes à l'égard de patients atteints de maladies neurologiques et psychiatriques. Par exemple, alors que plusieurs neuroscientifiques du début du vingtième siècle critiquaient le dualisme et soutenaient le monisme, le grand neurophysiologiste Charles Sherrington refusait d'en faire la promotion. Homme prudent et réfléchi, il croyait qu'étant donné les connaissances de son époque, cette thèse représentait une menace à la culture et aux valeurs humaines. ${ }^{89}$ Il y a des défis inhérents à la réflexion personnelle dans tous les domaines de recherche. Cependant, certaines approches telles que l'introduction d'une éducation aux sciences humaines et sociales dans la formation médicale (medical humanities) peuvent élargir la formation des chercheurs et des médecins et aider à disséminer les habiletés de réflexion autocritique.

\section{Conclusion}

\section{Un appel à la conscience morale inquiète}

En explorant les diverses facettes du fonctionnement normal et pathologique du système nerveux, les chercheurs en neurosciences participent au développement des soins de santé. En cela, ils contribuent à améliorer la qualité de vie de patients souffrant de maladies pour lesquelles il n'y a parfois encore trop peu d'options thérapeutiques. De plus, avec l'émergence de meilleures explications médico-scientifiques de la maladie mentale issues des neurosciences, les chercheurs dissipent les préjugés et la stigmatisation et contribuent donc à approfondir notre compréhension des personnes qui en souffrent.

La conduite responsable de la recherche comporte toutefois de multiples enjeux pour les chercheurs. Les exemples des découvertes fortuites en neuroimagerie, de l'usage améliorant de neurotechnologies ainsi que de la communication publique des résultats démontrent qu'il est nécessaire d'opter pour une vision large de la responsabilité des chercheurs. Nous espérons que le cadre multidimensionnelle de responsabilités neuroéthiques que nous avons proposé puisse alimenter la réflexion personnelle sur les enjeux éthiques inhérents aux neurosciences et une discussion sur le rôle des neuroscientifiques en tant que communauté. De fait, si les lignes directrices formelles en matière d'éthique aident le chercheur à conduire de manière éthique sa recherche, les chercheurs demeurent les piliers de la recherche éthique. Comme le bioéthicien Hubert Doucet ${ }^{90}$ a remarqué quant à la réflexion éthique en génétique, au-delà des codes et des lignes directrices, une conscience inquiète est peutêtre un tout aussi grand atout que des régulations qui peuvent étouffer la réflexion et la prise de conscience des responsabilités individuelles.

\section{RÉFERÉNCES}

1. Illes J, Racine E. Imaging or imagining? A neuroethics challenge informed by genetics. Am J of Bioeth. 2005; 5(2):5-18.

2. Abbott A. Deep in thought. Nature 2005; 436:18-9.

3. Ausman JI. I told you it was going to happen. Surg Neurol. 2004; 61:313-4.

4. Garland B. Neuroscience and the law: Brain, mind and the scales of justice. Washington, DC: The American Association for the Advancement of Science and The Dana Foundation; 2004.

5. Gura T. Big plans for little brains. Nature. 2005; 435:1156-8.

6. Editorial. Open your mind. The Economist; May 23, 2002.

7. Editorial. Sex, race and brain-scanning. The Economist; July 28, 2001.

8. Editorial. Brain scam? Nat Neurosci. 2004; 77:683.

9. Desmond JE, Chen SHA. Ethical issues in the clinical application of fMRI: factors affecting the validity and interpretation of activations. Brain Cogn. 2002; 50:482-97.

10. Illes J, Racine E, Kirschen MP. A picture is worth a thousand words, but which one thousand? In: Illes J, editor. Neuroethics: defining the issues in research, practice and policy. Oxford: Oxford University Press; 2005. p.149-68.

11. Katzman GL, Dagher AP, Patronas NJ. Incidental findings on brain magnetic resonance imaging from 1000 asymptomatic volunteers. JAMA. 1999; 281:36-9.

12. Kim BS, Illes J, Kaplan RT, Reiss A, Atlas SW. Incidental findings on pediatric MR images of the brain. AJNR Am J Neuroradiol. 2002; 23:1674-7.

13. Weber F, Knopf H. Incidental findings in magnetic resonance imaging of the brains of healthy young men. J Neurol Sci. 2006; 210:81-4. 
14. Central Brain Tumor Registry of the United States. Statistical report: Primary brain tumors in the United States, 1997-2001. Hinsdale, Il: Central Brain Tumor Registry of the United States; 2004.

15. Illes J, Desmond JE, Huang LF, Raffin TA, Atlas SW. Ethical and practical considerations in managing incidental findings in functional magnetic resonance imaging. Brain Cogn. 2002; 50:358-65.

16. Mazziotta JC. Window on the brain. Archives of Neurology 2000; 57:1413-21

17. Toga AW. Imaging databases and neuroscience. The Neuroscientist. 2002; 85:423-36.

18. Olson S. Brain scans raise privacy concerns. Science. 2005; 307:1548-50.

19. Editorial. Scanning the social brain. Nat Neurosci. 2003; 6:1239.

20. Pezawas L, Meyer-Lindenberg A, Drabant EM, Verchinski BA, Munoz KE, Kolachana BS, et al. 5-HTTLPR polymorphism impacts human cingulate-amygdala interactions: a genetic susceptibility mechanism for depression. Nat Neurosci. 2005; 8:828-34.

21. Hyman SE. Diagnosing disorders. Sci Am. 2003; Sep: 96-103.

22. Rosen AC, Bodke ALW, Pearl A, Yesavage JA. Ethical, and practical issues in applying functional imaging to the clinical management of Alzheimer's disease. Brain Cogn. 2002; 50:498519.

23. Illes J, Kann D, Karetsky K, Letourneau P, Raffin TA, SchraedleyDesmond P, et al. Advertising, patient decision making, and selfreferral for computed tomographic and magnetic resonance imaging. Arch Intern Med. 2004; 164:2415-9.

24. Illes J, Fan E, Koenig B, Raffin TA, Kann D, Atlas SW. Self-referred whole-body CT imaging: current implications for health care consumers. Radiology. 2003; 228:346-51.

25. Health Canada. Whole body screening using MRI or CT technology; http://www.hc-sc.gc.ca/iyh-vsv/med/mri-irm_e.html, accessed April 11, 2006.

26. US Food and Drug Administration. Whole body scanning using computer tomography (CT); http://www.fda.gov/cdrh/ct/, accessed April 11, 2006.

27. Cho MK. Conflicts of interest in magnetic resonance imaging: issues in clinical practice and research. Top Magn Reson Imaging. 2002; 13:73-8.

28. Canadian Institute for Health Information. Medical Imaging in Canada 2004. Ottawa: Canadian Institute for Health Information; 2004.

29. Racine E, Bar-Ilan O, Illes J. fMRI in the public eye. Nat Rev Neurosci. 2005; 6:159-64.

30. Check E. Ethicists urge caution over emotive power of brain scans. Nature. 2005; 435:254-5.

31. Bruer JT. The brain and child development: time for some critical thinking. Public Health Rep. 1998; 113:388-98.

32. Illes $\mathbf{J}$. Neuroethics in a new era of neuroimaging. AJNR Am J Neuroradiol. 2003; 24:1739-41.

33. Hilgenberg S. Formation, malformation, and transformation: my experience as medical student and patient. Stanford Med Student Clin J. 2005; 9:22-5.

34. Anonymous. How volunteering for an MRI scan changed my life. Nature. 2005; 434:17.

35. Greely H. Human genomics research: new challenges for research ethics. Perspect Biol and Med. 2001;44:221-9.

36. Philips M. Coping with unsuspected findings in volunteers. Nature. 2005; 434:17.

37. Wendler D. Risk standards for pediatric research: rethinking the Grimes ruling. Kennedy Inst Ethics J. 2004;14:187-98.

38. Illes J, Kirschen MP, Edwards E, Stanford LR, Bandettini P, Cho $\mathrm{MK}$, et al. Incidental findings in brain imaging research. Science. 2006; 311:783-4.

39. Elliott C. Pursued by happiness and beaten senseless: Prozac and the American dream. Hastings Cent Rep. 2000; 30(2):7-12.

40. Chatterjee A. Cosmetic neurology: the controversy over enhancing movement, mentation, and mood. Neurology. 2004; 63:968-74.
41. Farah MJ, Illes J, Cook-Deegan R, Gardner H, Kandel E, King P, et al. Neurocognitive enhancement: what can we do and what should we do? Nat Rev Neurosci. 2004;5:421-5.

42. Canadian Institute for Health Information. Drug Expanditure in Canada 1985 to 2004. Ottawa: Canadian Institute for Health Information; 2005.

43. Régie de l'assurance maladie du Québec. Portrait quotidien de la consommation médicamenteuse des personnes de 64 ans ou moins. Québec: Régie de l'assurance maladie du Québec; 2002.

44. Régie de l'assurance maladie du Québec. Portrait quotidien de la consommation médicamenteuse des personnes âgées non hébergées. Québec: Régie de l'assurance maladie du Québec; 2001.

45. Diller LH. The run on Ritalin: attention deficit disorder and stimulant treatment in the 1990s. Hastings Cent Rep. 1996; 26(2):12-8.

46. Elliott R, Sahakian BJ, Matthews K, Bannerjea A, Rimmer J, Robbins TW. Effects of methylphenidate on spatial working memory and planning in healthy young adults. Psychopharmacology. 1997; 131:196-206.

47. Mehta MA, Owen AM, Sahakian BJ, Mavaddat N, Pickard JD, Robbins TW. Methylphenidate enhances working memory by modulating discrete frontal and parietal lobe regions in the human brain. J Neurosci. 2000; 20:RC65.

48. Babcock Q, Byrne T. Student perceptions of methylphenidate abuse at a public liberal arts college. J Am Coll Health. 2000; 49(3):143-5.

49. Lynch G. Memory enhancement: the search for mechanism-based drugs. Nat Neurosci Supplement. 2002; 5:1035-8.

50. Rose SP. 'Smart drugs': Do they work? Are they ethical? Will they be legal? Nat Rev Neurosci. 2002; 3:975-9.

51. Yesavage JA, Mumenthaler MS, Taylor JL, Friedman L, O'Hara R, Sheikh J, et al. Donepezil and flight simulator performance: effects on retention of complex skills. Neurology. 2003; 59(1 of 2):123-5.

52. Pitman RK, Sanders KM, Zusman RM, Healy AR, Cheema F, Lasko $\mathrm{NB}$, et al. Pilot study of secondary prevention of posttraumatic stress disorder with propranolol. Biol Psychiatry. 2002; 51:18992.

53. Vastag B. Poised to challenge need for sleep, 'wakefulness enhancer' rouses concerns. JAMA. 2004; 291:167-70.

54. Foster RG, Wulff K. The rhythm of rest and excess. Nature Rev Neurosci. 2005; 6:407-44.

55. Blank RH. Brain policy: How the new neuroscience will change our lives and our politics. Washington, DC: Georgetown University Press; 1999.

56. Racine E. Thérapie ou amélioration? Philosophie des neurosciences et éthique des neurotechnologies. Ethica. 2002; 14(1):70-100.

57. Nicolelis MA. Actions from thoughts. Nature 2001; 409:403-7.

58. Schwartz AB. Cortical neural prosthetics. Annu Review Neurosci. 2004; 27:487-507.

59. Nicolelis MA. Brain-machine interfaces to restore motor function and probe neural circuits. Nat Rev Neurosci. 2003; 4:417-22.

60. Hetling JR, Baig-Silva MS. Neural prostheses for vision: designing a functional interface with retinal neurons. Neurol Res. 2004; 26:21-34.

61. Walter B, Vitek JL. Surgical treatment for Parkinson's disease. Lancet Neurol. 2004; 3:719-28.

62. Mayberg HS, Lozano AM, Voon V, McNeely HE, Seminowicz D, Hamani C, et al. Deep brain stimulation for treatment-resistant depression. Neuron. 2005; 45:651-60.

63. Maguire GQ, McGee EM. Implantable brain chips? Time for debate. Hastings Cent Rep. 1999; 29(1):7-13.

64. Hoag H. Neuroengineering: remote control. Nature. 2003; 423: 796-8.

65. Wickelgren I. Neuroscience. Tapping the mind. Science. 2003; 299:496-9.

66. Snyder AW, Mulcahy E, Taylor JL, Micthell JD, Sachdev P, Gandevia SC. Savant like skills exposed in normal people by suppressing the left fronto-temporal lobe. J Integr Neurosci. 2003; 22:149-58

67. Carey B. Implantable device for depression. The New York Times, May 21, 2005:A1. 
68. Hauser SL. The shape of things to come. Neurology. 2004; 63:94850

69. Flower R. Lifestyle drugs: pharmacology and the social agenda. Trends Pharmacol Sci. 2004; 25:182-5.

70. Allen DB, Fost N. hGH for short stature: ethical issues raised by expanded access. The J Pediatr. 2004; 144:648-52.

71. Gostin LO. Ethical considerations of psychosurgery: the unhappy legacy of the prefrontal lobotomy. J Med Ethics. 1980; 6:149-56.

72. Diefenbach GJ, Diefenbach D, Baumeister A, West M. Portrayal of lobotomy in the popular press: 1935-1960. J Hist Neurosci. 1999; 8:60-9.

73. Hagner M, Borck C. Mindful practices: on the neurosciences in the twenthieth century. Sci Context. 2001; 14:507-10.

74. Lanteri-Laura G. Examen historique et critique de l'éthique en neuropsychiatrie, dans le domaine de la recherche sur le cerveau et les thérapies. In: Huber G, editor. Cerveau et psychisme humains: quelle éthique? Paris: John Libbey Eurotext; 1996: p. 63-82.

75. Borck C. Electricity as a medium of psychic life: Electrotechnological adventures into psychodiagnosis in Weimar Germany. Sci Context. 2001; 14:565-90.

76. Rauscher FH, Shaw GL, Ky KN. Listening to Mozart enhances spatial-temporal reasoning: towards a neurophysiological basis. Neurosci Lett. 1995; 185:44-7.

77. Herculano-Houzel S. Do you know your brain? A survey on public neuroscience literacy at the closing of the decade of the brain. Neuroscientist. 2002; 82:98-110.

78. Racine E. Éthique de la discussion et génomique des populations. Éthique publique 2002; 41:77-90.

79. Tyers M, Brown E, Andrews DW, Bergeron JJ, Boone C, Bremner $\mathrm{R}$, et al. Problems with co-funding in Canada. Science. 2005; 308:1867.

80. Young SN. Universities, governments and industry: can the essential nature of universities survive the drive to commercialize? J Psychiatry Neurosci. 2005; 30:160-3.

81. Martinson BC, Anderson MS, de Vries R. Scientists behaving badly. Nature. 2005; 435:737-8.
82. Cho MK, McGee G, Magnus D. Lessons of the stem cell scandal. Science. 2006; 311:614-5.

83. Merali Z, Giles J. Databases in peril. Nature. 2005; 435:1010-1.

84. Illes J, Blakemore C, Hansson MG, Hensch TK, Leshner A, Maestro $\mathrm{G}$, et al. International perspectives on engaging the public in neuroscience. Nature Rev Neurosci. 2005; 6:977-82.

85. Zardetto-Smith A, Mu K, Phelps CL, Houtz LE, Royeen CB. Brains rule Fun $=$ learning $=$ neuroscience literacy. The Neuroscientist. 2002; 8:396-404.

86. People v. Weinstein. (591 N.Y.S. 2d 715). (Sup. Ct. 1992).

87. President's Council on Bioethics. Staff working paper: an overview of the impact of neuroscience evidence in criminal law; http://www.bioethics.gov/background/neuroscience_evidence.ht $\mathrm{ml}$, accessed August 18, 2005

88. Shevell MI. Neurosciences in the Third Reich: from ivory tower to death camps. Can J Neurol Sci. 1999; 26:132-8.

89. Smith R. Representations of mind: C.S. Sherrington and scientific opinion, c. 1930-1950. Sci Context. 2001; 14:511-29.

90. Doucet H. Le développement des morales, des législations et des codes, garder le dialogue ouvert et la conscience inquiète. In: Office des personnes handicappées du Québec, editor. Élargir les horizons: Perspectives scientifiques sur l'intégration sociale. Sainte Foy, Québec: Éditions Multimondes; 1994. p.135-41.

\section{REMERCIEMENTS}

La rédaction de cet article a été rendue possible grâce à une bourse postdoctorale du Conseil de recherches en sciences humaines du Canada et une subvention des National Institutes of Health/National Institute of Neurological Disorders and Stroke, R01 \#NS045831 (Judy Illes). Nous adressons des remerciements spéciaux à la Commission de l'Éthique, de la Science et de la Technologie (CEST) qui a financé une partie des travaux ayant conduit à cet article. 